\title{
Habiter en touriste, c'est habiter le Monde
}

Dwelling as a tourist means dwelling the world

\section{Olivier Lazzarotti}

\section{CpenEdition}

\section{Journals}

\section{Édition électronique}

URL : http://journals.openedition.org/tourisme/1484

DOI : $10.4000 /$ tourisme 1484

ISSN : 2492-7503

\section{Éditeur}

Éditions touristiques européennes

\section{Référence électronique}

Olivier Lazzarotti, « Habiter en touriste, c'est habiter le Monde », Mondes du Tourisme [En ligne], 14 |

2018, mis en ligne le 30 juin 2018, consulté le 23 septembre 2020. URL : http://

journals.openedition.org/tourisme/1484; DOI : https://doi.org/10.4000/tourisme.1484

Ce document a été généré automatiquement le 23 septembre 2020

\section{(c) (i) (9)}

Mondes du tourisme est mis à disposition selon les termes de la licence Creative Commons Attribution - Pas d'Utilisation Commerciale - Pas de Modification 4.0 International. 


\title{
Habiter en touriste, c'est habiter le Monde
}

Dwelling as a tourist means dwelling the world

\author{
Olivier Lazzarotti
}

1 «Il y a le ciel, le soleil et la mer... » et quelques douceurs de la voix suave d'un François Deguelt inspiré suffiraient presque à résumer ce qu'était la compréhension du tourisme au milieu des années 1960. Comme si le grand retournement héliotropique qui conduisit les touristes, après la Seconde Guerre mondiale, à choisir les eaux chaudes et les azurs ensoleillés, confirmait - encore et toujours - le lien fort entre les lieux touristiques fréquentés et les données physiques.

2 Pour autant, ces faisceaux d'explications fondés sur des évidences premières achèventils la réflexion? Cela dit, les remettre en cause ne revenait-il pas, réciproquement, à remettre en cause les catégories fondamentales d'une géographie encore marquée par ses vieilles régions : naturelles, avec ou sans guillemets, rurales, urbaines, etc., pour ne reprendre que quelques-unes des plus citées? Or, en traversant les lieux, les touristes, qui sont aujourd'hui des milliards, les interrogent. Et comment la géographie n'auraitelle pas dû, réflexivement, être également questionnée et ce, jusque dans ses fondamentaux?

3 Le concept d'habiter vient de là, de l'effervescence des années 1990 autour et avec la thématique touristique. Et si ses développements l'ont conduit au-delà du champ du tourisme, l'heure est peut-être d'y retourner, mais de son point de vue. À la fois bilan et perspective, le propos de cet article est donc de formuler un autre regard géographique sur ce phénomène sociétal qui, depuis deux siècles, présente la curieuse singularité d'être, à la fois, toujours identique et jamais le même. Toujours identique, parce que jamais le même? Alors, habiter en touriste : de quoi parle-t-on? 


\section{D'une géographie l'autre}

4 Au début des années 1990 (Lazzarotti, 2003), le tourisme avait bien sa géographie. Elle reposait alors sur une série de thèses d'État entreprises dans le courant des décennies 1960-1970. Parmi elles, et entre autres, sur la France, il y a celle de Daniel Clary (1977) sur la Normandie, celle de Jean-Michel Dewailly (1985) sur le littoral des départements du Nord et du Pas-de-Calais, celle d'Yvette Barbaza (1966) sur la Costa Brava, celle de Jacky Herbin (1980) sur le Tyrol, etc.

\section{A. Le tourisme, une géographie}

De substantiels matériaux avaient ainsi été rassemblés. Ils trouvèrent leur synthèse et leur fortune dans la publication de manuels (Lozato, 1985; Clary, 1993; Dewailly et Flament, 1993). Tout en marquant l'émergence du champ et, de ce point de vue, l'originalité de la géographie française aussi bien dans le champ international que disciplinaire, ils avaient posé les premiers jalons d'une géographie du tourisme, réciproque d'une timide sociologie du tourisme (Lanfant, 1972). Rapidement résumée, trois traits la caractérisaient alors. Le premier est celui de typologies qui reprennent les catégories physiques dominantes : montagnard, littoral, rural, le tourisme se plie aux critères de la régionalisation du moment. Un second est celui d'une prise en masse $d u$ phénomène: les touristes ne sont considérés que dans leur globalité, une globalité rapidement résumée par le terme usuel de "masse ». Troisième particularité, les définitions retenues pour leur approche comptable produisent une lecture essentiellement quantitative du tourisme, quand bien même les chiffres recueillis, dans des conditions d'observation toujours délicates, même aux frontières, laisseraient échapper une importante part du phénomène. La durée du séjour, essentiellement, fait critère : moins de 24 heures, et l'on est excursionniste; plus, on est touriste. Et, même si des travaux comme ceux de Georges Cazes (1992) engagent une approche plus thématique et plus théorique, ces quelques positions marquent le champ touristique, en partie comme la déclinaison d'une géographie aux lointains fondements.

\section{B. Contradiction(s)}

Bref, les études géographiques sur le tourisme, tout en présentant leurs spécificités, restent à l'image de la géographie du moment. Elles en portent donc logiquement quelques-unes des contradictions, à moins qu'elles ne finissent par les révéler. La plus lourde d'entre elles, peut-être, se présente comme la confrontation d'une science encore dominée par une approche sédentariste de la société. Elle privilégie l'immobilité et la stabilité. Dès lors, comment rendre compte des mouvements, des transformations géographiques et sociétales, avec des outils, des repères, si ce n'est une idéologie de nature fixiste? Comment comprendre qu'un phénomène en constant changement, inventant sans cesse de nouvelles pratiques, impliquant au passage de plus en plus de lieux, de plus en plus d'habitant(e)s, quitte, à l'occasion, à essuyer les critiques les plus répétées, peut aussi, et pour cela même, résister aux siècles qui passent pour se présenter aujourd'hui comme l'un des phénomènes sociaux les plus durables?

7 Ainsi, le tourisme éprouve la géographie des années 1990 par ses limites paradigmatiques : soit elle s'obstine à valoriser l'immobile et, dans ce cas, sa capacité à 
concevoir le phénomène touristique la laissera d'arrière-garde; soit elle ose le mouvement, mais alors elle devra se trouver prête à en payer le prix: celui d'un déplacement épistémologique. Car comment, en rompant avec le sédentarisme ambiant, inventer une science géographique capable de rendre compte des mouvements?

\section{Ouvertures}

8 La thématique de la mise en tourisme des lieux, celle de leur transformation par l'arrivée du tourisme, avait ouvert la voie (Dewailly et Flament, 1993). Elle renversait l'idée encore boueuse que les lieux sont figés de toute éternité, mais qu'ils naissent, changent et, parfois même, peuvent disparaître. Et elle suggérait l'idée qu'il pourrait y avoir un lien entre la mobilité des touristes et les changements des lieux. Mais ce sont sans doute les travaux de l'équipe MIT qui, du milieu des années 1990 à celui des années 2000, ont le plus contribué à faire basculer aussi bien les études sur le tourisme que les fondements épistémologiques de la géographie vers des positionnements autrement plus dynamiques. De manière embryonnaire, l'article collectif de 1997 (Knafou et al., 1997) pose ainsi les jalons du renversement. En suggérant de passer de la géographie du tourisme à l'approche géographique du tourisme, il engage un premier mouvement, celui d'une conception dimensionnelle de la géographie en particulier, donc des sciences sociales et humaines en général. Celles-ci ne se définiraient plus par les sujets propres de leurs études, mais par la singularité de leurs entrées dans ces sujets. Le tourisme, sujet global du champ des sciences sociales et humaines, aurait ainsi une entrée géographique, aussi bien qu'une entrée sociologique, anthropologique, philosophique, etc.

9 Comment, réciproquement, la question des lieux pourrait-elle ne pas être recentrée ? Voire reformulée ? Positivement, l'attribution de leur qualité « touristique » était liée à leurs « équipements " : hôtels, attractions diverses, paysages, etc. constituaient autant de critères observables et quantifiables, capables de définir un lieu touristique. Pourtant, rien n'est dit sur les gens qui les visitent, sur celles et ceux qui les occupent. La proposition qui est alors faite inverse le raisonnement : un lieu est touristique parce que les hommes et les femmes qui le fréquentent sont des touristes.

Ce n'est pas tout. «Le tourisme est [...] un changement d'habiter.» proposent également Rémy Knafou et al. (1997, p. 198). Le mot circulait. Il était « dans l'air ». Mais voilà qu'il se fixe dans l'écrit. Vu d'aujourd'hui, il suscite quelques commentaires. C'est qu'il fait partie du vocabulaire de la géographie classique. Il est porté par Paul Vidal (1903, p. 3) lui-même, comme signifiant de sa conception géographique de l'histoire. Son radical est repris par Maurice Le Lannou (1949, p. 11-12). Son « homme-habitant » vaut alors comme idéal politique de sa conception géographique du monde. Il et elle sont aussi critiqués par Pierre George, qui leur préfère l'approche marxiste de l'homme-producteur (Sivignon, 1993). L'habiter se trouve ainsi réexposé, réactivé. Mais, ce faisant, il est d'emblée détourné de son implicite idéologique dominant. Bouleversant la référence à l'homme immobile, dont la figure idéale est celle du paysan-soldat, le touriste est un habitant temporaire. Disons-le autrement : bien que temporaire, le touriste est aussi un habitant.

11 Revenu par le tourisme, le mot révèle alors toute sa richesse potentielle : comme mot d'usage courant, il est chargé de sens, de contradictions et d'usages multiples, ce qui en 
fait aussi sa richesse; comme mot chargé de tradition géographique, il s'ancre dans l'histoire intellectuelle, quelle qu'elle fut et quelles que soient les appréciations que l'on peut y porter ; comme concept à construire, il est suffisamment ouvert pour mettre en équivalence toutes les modalités d'habiter, des plus immobiles aux plus mobiles. En première analyse, il nomme ainsi, dans toutes ses possibilités, toutes ses virtualités, la dimension géographique de l'humanité; dans toutes ses modalités, soit l'infini combinaison des relations entre mobilités et immobilités.

Impulsée par le champ touristique, l'actualisation de l'habiter et sa re-conceptualisation ont désormais une vingtaine d'année. Elles sont passées par des voies qui ne doivent rien au tourisme. Désormais, le tourisme et l'habiter, comme concept et non comme expérience courante, peuvent faire bon ménage. Et ce sera l'occasion de se demander comment le travail fait sur l'habiter invite, aujourd'hui, à considérer ne serait-ce que quelques-unes des problématiques du champ touristique?

\section{Les touristes, des habitants}

13 Dès lors que l'on considère que c'est la présence des touristes qui fait la qualité touristique d'un lieu, l'approche géographique prend une nouvelle inflexion. Elle s'inscrit dans un plan plus anthropologique - comprenons ici l'épithète qui implique l'humain. Et du coup, la question des touristes, celle de leur définition, qui est aussi celle de leur compréhension, devient primordiale.

Or, produire une définition des touristes, c'est aussi se doter d'une notion capable de qualifier chacun et chacune. En considérant chaque homme ou chaque femme dans sa dimension géographique, la notion d'habitant(e) ouvre cette possibilité (Lazzarotti, 2006). Dès lors, le champ touristique peut devenir le terrain expérimental de cette notion d'habitant(e), comme l'occasion, tout à la fois, de l'éprouver et de la mettre en œuvre. Qu'est-ce donc qu'être touriste ? Et comment l'approche géographique peut-elle qualifier un habitant ou une habitante de touriste?

\section{A. Les touristes, des habitants déplacés}

Si tous les habitants mobiles ne sont pas des touristes, tous les touristes sont des habitants déplacés. Stricto sensu, ils changent de lieu. Quittant le lieu familier de leur résidence habituelle, quotidienne, ils font l'expérience d'autres lieux qui, à l'occasion, peuvent tout à fait être inconnus.

\section{Se déplacer, une compétence}

16 Cette expérience implique le franchissement de limites, de frontières. Certaines sont parfaitement visibles et balisées. D'autres n'apparaissent pas à l'œil nu, mais n'en sont pas moins importantes. Mais, dans tous les cas, leur "pente", pourrait-on dire, est toujours relative puisqu'elle résulte des relations entre les deux lieux. Parlant de " cultures », Claude Lévi-Strauss les considère comme des " écarts différentiels » (2002, p. 111). Plus orientées vers la géographie, on peut les qualifier d'«horizons géographiques » (Lazzarotti, 2006). En reprenant les études strictement touristiques, on peut en parler comme des «différentiels » (Équipe MIT, 2002). Mais peu importent les noms, pourvu qu'on garde l'idée, qui est que passer d'un lieu à un autre, c'est remettre 
en cause les savoirs qui permettent de se situer dans l'espace et le temps, donc parmi les autres. Si l'on peut considérer ces savoirs comme constitutifs d'un «capital géographique ", tout déplacement revient à une remise en cause de l'opérationnalité de ce capital. Dans tous les cas, se déplacer engage donc une prise de risque, aussi infime soit-elle. Le risque de la remise en cause de tout un ensemble de savoirs qui conditionnent l'habiter de chacun et chacune : question de langues, de nourriture, de monnaie, mais aussi de codes culturels, etc. Cela dit, passer d'un lieu à un autre relève donc d'une compétence, une compétence géographique (Cériani et al., 2004), en l'occurrence de déplacement (Lussault, 2007), tout près du sens de ce qu'Alain Tarrius a pu qualifier de « savoir-circuler» $(2000$, p. 124).

\section{Se déplacer, des confrontations}

L'expérience de l'habitant déplacé, dont le touriste est l'une des figures heureuses, est avec plus ou moins d'intensité celle d'une triple confrontation : une confrontation avec l'altérité, avec des lieux autres; une confrontation avec les autres ensuite; une confrontation avec soi-même, avec ses ignorances, ses doutes, parfois ses peurs enfin. Pour autant, tout ce qui fait la difficulté des touristes est peut-être aussi cela même qui fonde leurs pratiques: faire du tourisme, ce n'est pas seulement faire l'expérience de l'ailleurs, l'expérience de soi ailleurs, celles des autres, encore, là où l'on n'a pas l'habitude d'être ; faire du tourisme, c'est tout cela à la fois, et c'est tout cela qui donne chair à l'expérience d'une nouveauté.

Ainsi, le déplacement touristique aurait un sel particulier, une saveur souvent subtile, comprise entre l'excitation que procure la nouveauté, d'un côté, et le confort des lieux dont les mesures sont déjà connues, de l'autre.

\section{B. Apprendre pour être libre}

19 Maîtrisant partiellement mais toujours mieux les techniques géographiques de déplacements, les touristes accèdent ainsi à un degré de liberté augmenté dans le choix des lieux qu'ils fréquentent. Ils gagnent en autonomie. Faire un déplacement touristique, c'est évaluer les avantages et les coûts, les envies et les risques de telle ou telle destination. De fait, l'action résulte d'un équilibre, d'un argumentaire et, au final, d'une décision. Faire du tourisme, c'est ainsi faire des choix : aller ici ou là ? Pourquoi et comment?

Saisir les lieux touristiques comme des lieux «élus» par les habitants-touristes ne revient pas pour autant à considérer que les touristes sont des habitants absolument libres, décidant souverainement des termes de leurs envies. Comme tous les habitants, les touristes subissent des «contraintes » extérieures : il y a des lieux plus ou moins accessibles, que ce soit pour des raisons techniques ou sociétales. Les touristes sont en outre porteurs de limites propres à chacun et chacune: question économique, par exemple, mais aussi cognitive et, le cas échéant, psychologique.

Cela dit, il faut également constater que la part grandissante de liberté des mouvements touristiques s'accroît à mesure qu'elle s'apprend. Savoir se déplacer, apprendre à être dans un lieu inconnu sans y être perdu, c'est aussi cela faire du tourisme. Il n'existe pas de cours pour cela. Mais des guides, sur place, que l'on peut considérer comme des "passeurs d'altérités " (Équipe MIT, 2002). Ces médiateurs sont 
capables d'initier les touristes aux lieux qu'ils découvrent. Ils montrent, expliquent, parlent. De tels guides existent aussi, et depuis longtemps, sous forme de livres. Ils produisent leur carte des "hauts lieux ", indiquent les manières de s'y rendre, d'y vivre, parfois d'y survivre. Faire du tourisme relève ainsi d'apprentissages et produit des apprentissages. Et, d'expérience en expérience, les apprentissages évoluent, ne serait-ce qu'en se transmettant. Les générations se suivent, mais ne pratiquent pas le tourisme de la même manière. Les modèles réputés et fonctionnels qui invitaient le plus grand nombre à des séjours peu chers ont marqué leurs époques. On peut penser au cas tunisien. Les touristes formés, habitués parce que venus et revenus, à Djerba par exemple, se tournent ensuite vers de nouvelles expériences, plus personnelles peutêtre, si ce n'est personnalisées. Le tourisme change donc, car les touristes changent en se renouvelant.

Il convient désormais de le reconnaître : en dehors de toute apparence, le tourisme est donc une pédagogie, une pédagogie pratique, puisqu'elle s'apprend en partie in situ, qu'elle s'apprend «en se faisant». Une pédagogie du Monde, puisqu'elle porte, précisément, sur les lieux du Monde.

\section{Des habitants : pratiquer, imaginer}

Par leurs faits et gestes, les habitants-touristes mettent en œuvre des pratiques. On peut ici reprendre la définition d'une pratique de Michel Foucault : «[...] ce qu'ils [les hommes] font et comment ils le font.» (2004, p. 83). Il faudrait sans doute y ajouter "où » ils le font. Les pratiques peuvent ainsi être définies comme des actions géographiquement orientées, c'est-à-dire des actions qui prennent sens par rapport aux lieux où elles se déroulent. Plus particulièrement que tout autre phénomène social, le tourisme est donc si ce n'est une pratique, du moins un ensemble de pratiques. L'expression courante du français ne s'y trompe du reste pas: on est touriste parce qu'on fait du tourisme. Être touriste, c'est donc avoir des pratiques touristiques. Parmi elles, le repos, le jeu, la découverte, ont pu être considérés comme caractéristiques (Stock, 2003), même s'ils ne sont pas exclusifs des pratiques touristiques, en particulier parce que celles-ci ne cessent de se transformer, pour se diffuser aussi bien que s'inventer. Le lien contemporain entre les pratiques touristiques et les déambulations commerciales, par exemple, pourrait bien constituer une nouvelle catégorie qualificative du tourisme ou, plus précisément, de pratiques touristiques. Cela dit, les pratiques ne font pas tout.

Par leur présence, les touristes changent les lieux qu'ils parcourent. En l'occurrence, pratiques et regards sont liés. Être touriste, c'est aussi voir le Monde d'une certaine manière. De ce point de vue, les travaux d'Alain Corbin (1988) ont permis de saisir ce retournement des regards qui a fait des littoraux, lieux vides et inquiétants, des lieux de vacances enchantées pour des urbains en mouvement. En les voyant d'un autre point de vue, avec d'autres regards, les touristes transforment ainsi les lieux. L'importance du regard dans le tourisme trouve également son écho dans les productions des artistes les plus reconnus, attirés par la visibilité parfois criante de modernité du phénomène. On pense ici aux «plages de Trouville ", peintes par Claude Monnet. Mais l'esthétique touristique, ce sont aussi les imaginaires architecturaux dont le vocabulaire - ici militaire, là religieux, coloré et fantaisiste - édifie face aux éléments, parfois venteux et pluvieux, des habitations dont on ne sait pas toujours bien 
si elles relèvent de la catégorie des villas individuelles ou des immeubles collectifs urbains.

\section{Le tourisme, des cohabitations}

Le tourisme est rencontres, échanges. Il est commerce au sens le plus classique donc le plus large du terme : celui qui reconnaît une personne par son commerce agréable. Et ces rencontres sont multiples : rencontres entre les touristes eux-mêmes; rencontres entre les touristes et les autres habitants des lieux.

Habitants, les touristes sont donc aussi des cohabitants. Du reste, et dans tous les cas, au-delà même du renouvellement saisonnier des cohabitants-touristes, le tourisme est cohabitations. Il met en cause les interrelations humaines dans leur dimension géographique. Des interrelations dont les lieux ne sont pas les décors, mais les enjeux. $\mathrm{Et}$, même si cette lecture du phénomène n'est pas la plus courante, la cohabitation touristique se présente comme un modèle politique. Un modèle politique original, ne serait-ce que parce qu'il dure depuis maintenant près de deux siècles, alors même que ses cohabitants se renouvellent constamment. Un modèle, donc, qui se présente sous les traits un peu paradoxaux d'une " révolution durable ", pour reprendre les termes de l'équipe MIT (2011). Et cette situation est d'autant plus surprenante que le tourisme est aussi associé à une prise de liberté et que cette émancipation semble en contradiction avec l'idée même de faire modèle, $a$ fortiori au sens politique du terme. Un modèle, en effet, ne se définit-il pas au moment où il se reproduit?

\section{A. Baliser les lieux}

De fait, manifestée par les touristes cette liberté pourrait bien se révéler plus organisée, plus contrôlée qu'il n'y paraît. Du reste, il n'a pas été nécessaire d'attendre très longtemps pour assister à la mise en place de ce contrôle. Ainsi, les premières stations touristiques balnéaires rendent possibles les pratiques et les rencontres touristiques tout en les surveillant de très près. Les aménagements de l'espace y participent prioritairement. La plage et les premiers mètres de la mer sont rapidement balisés par des bouées et tout un ensemble de traits qui signalent, en les partageant, les zones de bains et les autres. C'est là que l'on peut désormais se baigner. Là, et pas ailleurs. La station elle-même est rigoureusement organisée selon des règles d'usage relativement strictes. Les nombreuses cartes postales sont là aussi pour le rappeler : même dans un lieu touristique, on ne fait pas n'importe quoi n'importe où.

Est-ce parce que les pratiques du corps, au cœur de ces pratiques touristiques, flirtent avec les sexualités qu'elles constituent l'un des grands enjeux des normes touristiques? Dès lors, il n'est pas étonnant que, plusieurs fois dans l'histoire, la question des vêtements ait fait problème, que ce soit pour se vêtir ou se dévêtir. Y compris dans le champ du tourisme donc, il y a les pratiques légitimes, acceptées, tolérées. Mais il y en a aussi d'autres, interdites voire prohibées. La cohabitation touristique, marque qualifiante des lieux touristiques, repose ainsi sur des ordres, des règles, des codes. Ils sont régis par les tenants des lieux, suivis ou contestés par les touristes qui, à l'occasion, peuvent aussi les changer.

À leurs manières, les parcs à thème sont les lieux qui ont sans doute poussé le plus loin le contrôle de tous. L'expérience des touristes aussi bien que l'établissement de leurs 
relations y sont "totalement " pris en charge. Cela commence par le rigoureux dessin du plan lui-même et se prolonge par l'organisation des attractions, y compris les modes et les temps d'attentes, par la largeur des rues qui décide des débits optimaux des flux d'habitants, etc. Tous ces éléments visent, le plus souvent avec succès, à un double but convergent : le premier est de satisfaire les visiteurs; le second est de les surveiller. De ce point de vue, les parcs à thème constituent probablement les premières expériences de gestion, si ce n'est de contrôle, des foules. À leurs manières et sans doute paradoxalement, ils ont pu constituer les laboratoires de techniques mises en œuvre dans les villes « dissuasives » (Terrolle, 2004) contemporaines.

Dire que les touristes sont des cohabitants, c'est donc reconnaitre la dimension politique du tourisme, ou, plus exactement, reconnaître le tourisme dans sa dimension politique. Non seulement la pratique et ses lieux mettent en cause les interrelations humaines mais ils les inscrivent dans des schémas fermes. Cela n'est pas spécifique au tourisme mais apparaît peut-être mieux dans le cas de cette activité. Peu importe, dès lors, que l'on soit à la montagne ou à la mer. Les paysages pourraient aussi bien n'être que "seconds», ce qui ne veut pas dire secondaires (Lussault, 2015), dans ce qui constitue le phénomène touristique. Entre le licite et l'interdit, entre ce qui libère et ce qui contraint, l'invisible faiseur du tourisme tient à son modèle politique.

\section{B. Des machines économiques}

31 Les lieux touristiques ont aussi leur économie. Leur durabilité dépend en effet, pour partie, de la production de richesses qui s'y opère. Victor Hugo (1967), peut-être le premier, puis Frederick Law Olmsted (1865) dans son rapport préliminaire pour le classement du parc du Yosemite, y sont revenus pour justifier l'ouverture au public des " monuments ». Leur mise en tourisme est le moyen le plus sûr pour leur assurer une rentabilité, finalement le meilleur gage de leur conservation face aux appétits financiers des exploitants en tout genre.

C'est donc que le tourisme peut être un commerce rentable (Lazzarotti, 2008). Une première source de revenu est la spéculation foncière. La construction touristique d'un lieu mobilise des entreprises en tout genre, que stimule la demande de logements. Et même si cette phase ne dure jamais très longtemps, elle peut être très active. Les premiers temps d'une station sont souvent ceux d'une ville nouvelle, parfois même d'une ville-champignon. Et tant que la demande est plus forte que l'offre, la machine tourne. Puis, cela fait, par leur présence, les touristes dépensent. Ils alimentent alors un autre type de ressources, celles d'une "économie présentielle» (Davezie, 2008) soutenue, autant que faire se peut, par toutes les arguties incitatives possibles.

Dès lors, c'est la ressource des touristes que se disputent désormais les lieux touristiques. Et si la ressource est chaque année un peu plus abondante, les lieux touristiques pourraient bien aussi être toujours un peu plus nombreux. La concurrence entre les lieux touristiques bat ainsi son plein et ce, dans des termes d'autant plus renouvelés que les compétences de mobilité, donc le degré de liberté des touristes, grandissent. Pour faire venir les touristes, les tenants des lieux doivent désormais travailler à leur double qualité. La première est celle de leur singularité. Donner envie de venir est une chose. Mais le mieux est que l'offre soit ici et pas ailleurs, selon une logique que définit le terme de « rente de monopole ( Harvey, 2001). Il est difficile de voir Dubaï sans y aller (Schmid, 2009). Mais, pour être singulière, l'offre n'en doit pas 
moins être accessible. Cela passe alors par la connexion du lieu aux grands moyens de transports du Monde.

Cela dit, la possibilité technique de transport ne suffit pas toujours. Car ces lieux doivent aussi permettre à tous et à chacun de s'y retrouver. La singularité, l'étrangeté même, doivent ainsi être combinées avec des traits courants, si ce n'est communs, qui font que ceux qui n'y sont jamais venus peuvent tout de même s'y rendre. Idéalement, il faut que les touristes soient dépaysés sans toutefois être perdus. La fragile dynamique des lieux touristiques repose ainsi sur une délicate alchimie entre l'étrange et le familier, entre le loin et lointain (Lazzarotti, 2001). Géographiquement parlant, elle est, à la fois, celle du local et du mondial. Trop local, trop étrange, etc. et les touristes ne viendront pas, ou peu nombreux. Trop banal, trop accessible, et ils ne viendront pas non plus. Le choix de la «bonne distance » est ainsi l'une des raisons de la vitalité des "mémoires-Monde", où les mémoires localisent ce que le tourisme mondialise (Lazzarotti, 2012).

\section{Une cohabitation pacifique}

Indépendamment de tous les types de pratiques et de leurs diversifications, de tous les types d'habitants et de tous les types d'enjeux économiques, le tourisme est une manière de vivre ensemble. Ponctuellement et temporairement, il est une manière d'organiser la vie collective de gens qui ne se connaissent pas mais qui, de fait, cohabitent. Or, ce qui est peut-être le plus significatif, c'est que ces cohabitations se font, en dépit des différences entre les cohabitants, de manières pacifiques. En cela, ce modèle se distingue d'autres, plus violents, parfois meurtriers.

\section{Différents, mais ensemble}

36 Les premières différences sont celles qui distinguent les touristes eux-mêmes. Venus de plus ou moins loin, parlant à l'occasion des langues différentes, ils se retrouvent les uns en face des autres. Pourtant, deux dispositions rendent possibles leurs rencontres avant même qu'elles ne se passent et, peut-être, au-delà même du renouvellement des protagonistes. La première est que tous ont décidé d'être là. Ils ont fait un même choix, même s'ils l'ont fait pour des raisons différentes. La seconde, qui découle en partie de la première, est qu'ils partagent, globalement, la même conception du lieu et de ses usages. Sans se connaître, et même s'ils peuvent venir d'horizons différents, les touristes se retrouvent là où ils sont comme dans un lieu commun, et s'y reconnaissent.

Les secondes différences ont très souvent été décrites comme celles existant entre les résidents du lieu et les habitants temporaires que sont les touristes. La plupart du temps, elles sont présentées comme des oppositions, à l'occasion radicales. Les touristes, éventuellement décrits comme des envahisseurs peu scrupuleux, bouleverseraient les sociétés locales, qui en perdraient leurs équilibres et leur « identité ». Pourtant, il semble que cette lecture soit trop simple, pour ne pas dire trop simpliste. D'une part, comme le souligne Philippe Violier (2008: 69), il n'est pas toujours aisé de définir les membres d'une société locale : le «local» est-il celui qui réside, disons à titre principal ? Celui qui vote ? Celui qui est né là, même s'il n'y réside plus? L'ancien vacancier venu s'installer? D'autre part, les relations aux touristes ne sont pas toujours homogènes. Certains « locaux » participent à la mise en tourisme : ils ont des commerces, hôteliers par exemple mais pas seulement; ils sont loueurs de 
biens et, plus généralement, participent à l'économie touristique. Il est donc difficile de considérer les sociétés locales comme uniformes. Il est encore plus rare de constater que les lieux ont été mis en tourisme contre leur volonté. Les cas de Baie-Saint-Paul et de La Malbaie au Québec en offrent une belle illustration. Les deux lieux sont assez comparables: même localisation, sur la rive gauche du Saint-Laurent, à une quarantaine de kilomètres de Québec; même paysage face au fleuve, etc. Pourtant, si les «locaux» de La Malbaie se sont précocement ouverts au tourisme, ceux de BaieSaint-Paul l'ont pendant longtemps refusé. La différence entre le premier lieu, mis en tourisme, et le second, longtemps resté en dehors, tient donc aux "locaux» (Lazzarotti, 2001). Finalement, il n'y a guère de mise en tourisme qui ne soit aussi soutenue par au moins une partie des membres des sociétés « locales ». Et les élections municipales sont, régulièrement, l'occasion de faire le point sur la question, en particulier quand le tourisme est un débat politique.

\section{Quels conflits pour quelle cohabitation?}

Pour toutes ces raisons, il semble difficile de considérer de manière homogène les habitants-touristes aussi bien que les résidents d'un lieu. Pour autant, la question des relations de cohabitation entre ce qui serait deux catégories d'habitants n'en demeure pas moins exprimée, en particulier en termes quantitatifs. L'argumentaire est souvent répété. La question n'est pas celle du tourisme, mais celle de la présence de touristes jugés «trop » nombreux. Pourtant, la question des limites quantitatives n'est jamais énoncée, et personne ne dit à partir de combien de touristes on est dans le "trop ", pas plus que la manière exacte dont on compte. Cela dit, toutes ces difficultés, qui sont aussi bien théoriques qu'opérationnelles, ne font peut-être que mettre en évidence que l'aspect quantitatif n'est pas le seul en jeu.

39 Cohabiter, c'est en effet aussi se positionner par rapport à un ensemble d'ordres, de règles, de normes qui participent à la définition du lieu : qu'y fait-on? Comment ? Où ? Voilà autant de questions, parmi d'autres, qui fondent les bases locales du vivreensemble. Dès lors, et parfois peut-être au-delà des différences entre tous les habitants d'un lieu, ce qui se joue, c'est la nature même de l'ordre dominant et, dans cette logique, la place qui est faite au tourisme tout autant que celle des touristes euxmêmes. Ce qui entre donc en contradiction, et à l'occasion en conflit, ce ne sont pas tant des habitants - touristes ou «locaux»- que le regard qu'ils portent et les pratiques qu'ils mettent en œuvre dans le lieu qu'ils habitent. De tels conflits portent ainsi sur les ordres locaux, ceux qui conditionnent les interrelations humaines qui s'y déroulent. Ce qui se joue, c'est donc au moins autant un conflit de légitimité que de nombre, celui du droit au lieu autant que du droit des lieux (Lazzarotti, 2006). La question de la légitimation des présences prend ici une importance particulière. Du droit du premier occupant ou de celui du dernier arrivé, lequel prévaut? Du droit de l'habitant permanent ou de celui de l'habitant de passage, lequel s'impose ? De ce point de vue, la problématique touristique de la cohabitation entre différents habitants n'est que la formulation particulière d'un débat plus général: celui entre un régime de cohabitation ouvert, faisant une place à l'autre, ou bien fermé, replié sur lui-même. C'est ainsi, bien au-delà de lui-même, que le tourisme mérite d'être considéré.

En effet, phénomène marginal des sociétés de la Première Révolution industrielle, le tourisme connaît aujourd'hui une extension sans précédent, celle d'une "troisième révolution touristique» qui gagne en ce début de siècle des pans entiers de pays 
récemment peu touristiques. Mieux - ou pire ?-, ces nouveaux horizons du tourisme sont en train de se hisser au rang des pays les plus touristiques. La Chine, à elle seule, compte à peu près autant de touristes qu'il y a de touristes internationaux recensés par l'Organisation mondiale du tourisme (OMT). Et, dans les deux cas, cela dépasse le milliard. On peut dès lors se poser la question: le Monde entier n'est-il pas en train d'être façonné sur le modèle d'une vaste aire touristique ? Et la question pourrait alors être reformulée : le Monde contemporain n'est-il pas en train de se construire selon des pratiques, des règles, des normes inventées et éprouvées par et avec le tourisme?

41 La multiplication des déplacements touristiques croise la diversification de toutes les mobilités et l'infinité de leurs combinaisons possibles. Si les États-nations se sont construits sur l'idéal politique de la sédentarité, avec comme habitant modèle le paysan-soldat, le Monde contemporain est celui de l'émergence des «sociétés à habitants mobiles ». Dès lors, les pratiques touristiques rejoignent une dynamique beaucoup plus ample que celle de leur seul champ. Le tourisme devient ainsi l'un des éléments d'une tendance beaucoup plus vaste. Dès lors, les problématiques rencontrées dans le tourisme se posent aussi comme celles du Monde qui s'avance: l'habitant, formé et compétent, renforce à chaque déplacement son autonomie géographique ; comment dès lors concevoir, reconnaître, légitimer, valider, etc. la place des uns et des autres en régime d' ' habiter » mobilitaire ? Un habitant mobile, fût-il un touriste, est-il un habitant sans droit politique, sans autre droit, tout de même, que celui de dépenser son argent?

La problématique est d'autant plus importante que la formation du Monde contemporain pourrait bien être en train d'absorber le tourisme. Et Michel Lussault (2007), annonçant la fin de sa spécificité, n’a-t-il pas proposé de le considérer comme un « genre commun»?

Cela voudrait-il dire que le tourisme ne serait plus un phénomène en soi, isolable du reste de la société et que chacun, y compris sans se déplacer, sans rupture entre quotidien et hors-quotidien, pourrait être touriste "chez lui », en quelque sorte ? À sa manière, Emmanuelle Peyvel (2016) propose l'idée de «degré de touristicité » des habitants, comme la part de ce qu'il y a de touristique en chacun. Frédéric Darbellay et Mathis Stock (2012) proposent, quant à eux, de passer du tourisme au touristique, suggérant que le tourisme n'est pas un monde social autonome afin de constater les multiples formes d'hybridation d'un "secteur touristique " de plus en plus associé à d'autres, y compris le travail.

\section{Du tourisme et de son « habiter »}

Reconceptualisé à partir du champ touristique en bouleversement au cours des années 1990 en confirmant son sens bien au-delà de l'idée de se loger, l'habiter est désormais reconnu comme l'un des concepts géographiques les plus pertinents de ce début de siècle: de nombreux ouvrages, d'horizons variés, l'affichent en titre $^{1}$; des colloques $^{2}$ et des séminaires de recherches, au-delà même de la géographie ${ }^{3}$, lui sont consacrés; des articles ${ }^{4}$ mais également des numéros thématiques de revue ${ }^{5}$ en traitent ; le Festival de Saint-Dié de 2014 participa à sa diffusion et les programmes du second degré en font une charnière pédagogique ${ }^{6}$; d'autres ouvrages y font encore explicitement référence ${ }^{7} .$. 

interprétatives qui sont aussi, espère-t-on, quelques perspectives de recherche. D'abord, minimiser les vieilleries géographiques des catégories classiques n'a d'intérêt que comme point de départ d'un renouvellement problématique : en quoi être touriste alimente-t-il la dynamique propre de chaque habitant? Qu'apprend-on en tant que touriste et en quoi ce qui est appris change celui qui apprend ? La question de l'habitant est existentielle. Elle est politique, aussi, comme celle de la cohabitation, de son modèle politique. L'invisible du tourisme, ce qui se transmet dans ses lieux, ses ordres, ses pratiques, ses esthétiques même, structure un « régime d'habiter ", invisible dans son essence, mais fortement visible dans ses manifestations.

De ce fait, malgré des apparences changeantes, ce régime d'habiter est durable. N'est-il pas en train de devenir un modèle dominant qui attesterait, tout à la fois, de la formidable promotion du tourisme et de son infusion dans l'ensemble des sociétés? Dans l'alternative positive, une réponse à la question initiale pourrait être celle-ci : si le tourisme dure, c'est qu'au-delà de ses innombrables manifestations, il porte en lui les fondements de quelques dynamiques du Monde contemporain. Et si ce régime d'habiter touristique, d'abord laboratoire du Monde d'aujourd'hui, en devenait la norme? Autrement dit, et pour conclure, cette proposition conduirait alors à reconnaître qu'habiter en touriste, c'est habiter le Monde.

\section{BIBLIOGRAPHIE}

Yvette BARBAZA, Le paysage humain de la Costa Brava, Paris : Armand Colin, 1966.

Georges CAZES, Fondements pour une géographie du tourisme et des loisirs, coll. " amphi-géographie », Bréal, 1992.

Georges CAZES, Les nouvelles colonies de vacances ?, 2 tomes, L'Harmattan, 1989, 1992.

Georgia CÉRIANI, Rémy KNAFOU et Mathis STOCK, « Les compétences cachées du touriste », Sciences humaines, $\mathrm{n}^{\circ} 145,2004$.

Daniel CLARY, Le tourisme dans l'espace français, coll. « le géographe », Masson, 1993.

Daniel CLARY, La façade littorale de Paris, Ophrys, 1977.

Alain CORBIN, Le territoire du vide. L'Occident et le désir du rivage, 1750-1840, coll. « Historique », Aubier, 1988.

Frédéric DARBELLAY et Mathis STOCK, « Penser le touristique : nouveau paradigme ou interdisciplinarité ? », EspacesTemps.net, 2012 [http://www.espacestemps.net/articles/penserle-touristique-nouveau-paradigme-ou-interdisciplinarite/]

Laurent DAVEZIES, La République des territoires. La circulation invisible des richesses, coll. « La République des idées », Seuil, 2008.

Albert DEMANGEON, Problèmes de géographie humaine, Armand Colin, 1943. 
Jean-Michel DEWAILLY, Tourisme et loisirs dans le Nord-Pas-de-Calais. Approche géographique de la récréation dans une région urbaine et industrielle de l'Europe du Nord-Ouest, 2 tomes, Société de géographie de Lille, 1985.

Jean-Michel DEWAILLY et Émile FLAMENT, Géographie du tourisme et des loisirs, SEDES, 1993.

Équipe MIT, Tourismes 1, lieux communs, coll. « Mapemonde », Belin, 2002.

Équipe MIT, Tourismes 3, la révolution durable, coll. « Mapemonde », Belin, 2011.

Michel FoucAult, Qu'est-ce que les Lumières ?, coll. « La philothèque », commentaires de Olivier DECKENS, Bréal, 2004.

David HARVEY, Géographie de la domination, Les prairies ordinaires, 2001.

Jacky HERBIN, Le tourisme au Tyrol autrichien, 2 tomes, Éditions des cahiers de l'Alpe, 1980.

Victor HUGO, « Guerre aux démolisseurs! », dans Victor HUGo, CEuvres complètes, Tome IV, Club français du livre, 1967.

Rémy KNAFOU, Mireille BRUSTON, Florence DEPREST, Philippe DUHAMEL, Jean-Christophe GAY et Isabelle SACAREAU, « Une approche géographique du tourisme », Espace Géographique, n 3, 1997.

Marie-Françoise LANFANT, Les théories du loisir. Sociologie du loisirs et idéologie, coll. « Le sociologue », PUF, 1972.

Olivier LAZZAROTTI, Des lieux pour mémoires, coll. « Le temps des idées », Armand Colin, 2012.

Olivier LAZZAROTTI, « Wimereux, station balnéaire de villégiature (1860-1930) : La "machine à habiter" ", In Situ, revue en ligne de l'inventaire, ministère de la culture, n 9, 2008 [http:// www.revue.inventaire.culture.gouv.fr/insitu/insitu/article.xsp? numero=9\&id_article=lazzarotti-578]

Olivier LAZZAROTTI, Habiter, la condition géographique, coll. « Mappemonde », Belin, 2006.

Olivier LAZZAROTTI, «L'horizon géographique ou conditions pour des rencontres heureuses », dans Noël BARBE et Emmanuelle JALLON (dir.), Migrations, itinérances et mobilités, actes des journées d'études de Vesoul, 8-10 mars 2006, Vesoul : Conservation départementale des musées de HauteSaône, 2008.

Olivier LAZZAROTTI, «About French Tourism Geographies: a review », Tourism Geographies, nº 4 (2), 2002.

Olivier LAZZAROTTI, « Tourismus: Von Orten und Menschen », Voyage, Jahrbuch für Reise \& Tourismusforschung, 2001.

Olivier LAZZAROTTI, «Je me souviens : recherches au pays où le passé fait des lieux. Carnet de voyage ", Cahiers de Géographie du Québec, vol. 45, n 124, 2001.

Maurice LE LANNOU, La géographie humaine, coll. « Bibliothèque de Philosophie scientifique », Flammarion, 1949.

Claude LÉVI-STRAUSS, Race et histoire. Race et culture, coll. « Bibliothèque Idées ", Albin MichelUnesco, 2002.

Jean-Pierre lozATo-GIOTARD, Géographie du tourisme, coll. « Géographie », Masson, 1993, 4e éd.

Michel LusSAUlt, « L'expérience de l'habitation », dans Olivier LAZZAROTTI, Béatrice COLLIGNON et Philippe PELLETIER (dir.), Habiter : mots et regards croisés, Annales de géographie, n 704, 2015. 
Michel LusSAUlt, L'homme spatial. La construction sociale de l'espace humain, coll. « La couleur des idées », Le Seuil, 2007.

Michel Lussault, « Le tourisme, un genre commun », dans Philippe DuHAMEl et Rémy KNAFou (dir.), Mondes urbains du tourisme, coll. « Mappemonde », Belin, 2007.

Frederick Law OLMSTED, Yosemite and the Mariposa Grove, a preliminary report, 1865 [disponible en ligne : http://www.yosemite.ca.us/library/olmsted/report.html]

Emmanuelle PEYVEL, L'invitation au voyage. Géographie postcoloniale du tourisme domestique au Viêt Nam, coll. « De l'Orient à l'Occident », ENS Éditions, 2016.

Heiko SCHMID, Economy of fascination. Dubai and Las Vegas as themed urban landscapes, «Urbanisierung der Erde, 11 », Gebrüder Borntraeger Verlagsbuchhandlung, 2009.

Michel sivignon, « Du verbe habiter et de son amère actualité », Revue de géographie de Lyon, vol. 68, n 4, 1993.

Mathis sтоск, « L'hypothèse de l'habiter poly-topique : pratiquer les lieux géographiques dans les sociétés à individus mobiles », EspacesTemps.net, Textuel, 2006 [http://espacestemps.net/ document1853.html]

Mathis sтоск, Le tourisme. Acteurs, lieux et enjeux, coll. « Belinsup. Géographie », Belin, 2003.

Alain TARRIUS, Les nouveaux cosmopolitismes. Mobilités, identités, territoires, coll. « Essai », l'Aube, 2000.

Daniel TERROLLE, « La ville dissuasive : l'envers de la solidarité avec les SDF », Espaces et sociétés, n 116-117, 2004 [DOI : 10.3917/esp.116.0143 ; http://www.cairn.info/revue-espaces-etsocietes-2004-1-page-143.htm]

Paul VIDAL DE LA BLACHE, Tableau de la géographie de la France, Armand Colin, 1903.

Philippe VIOLIER, Tourisme et développement local, coll. « Belinsup. Tourisme », Belin, 2008.

\section{NOTES}

1. Par exemple: Fiona MEADOws (dir.), Habiter le campement, Arles et Paris: Actes Sud, Cité de l'architecture et du patrimoine, Hors-série L'impensé, 2016 ; Pierre GIORGINI, Au crépuscule des lieux. Habiter ce monde en transition fulgurante, Bayard, 2016 ; Jean-Marc BESSE, Habiter, un monde à mon image, coll. "Sens Propre», Flammarion, 2013; Olivier LAZZAROTTI, Habiter, la condition géographique, coll. «Mappemonde», Belin, 2006 ; George-Hubert de RADKOWSKI, Anthropologie de l'habiter. Vers le nomadisme, PUF, 2002 ; etc.

2. Voir par exemple : "L'Homme habite en touriste ", Troisièmes rencontres de l'habiter, SaintRiquier, septembre 2016.

3. Voir par exemple: «Habiter. L'Encrage en littérature contemporaine », ENS Ulm, janvierseptembre 2016, etc.

4. Voir par exemple: André-Frédéric HoYAux, «Les constructions des mondes de l'habitant: éclairage pragmatique et herméneutique », Cybergeo: European Journal of Geography, Épistémologie, Histoire de la Géographie, Didactique, document 232, 2003 [http://cybergeo.revues.org/3401 ; DOI : 10.4000/cybergeo.3401] ; Mathis STOcK, «L'hypothèse de l'habiter poly-topique : pratiquer les lieux géographiques dans les sociétés à individus mobiles », EspacesTemps.net, Travaux, 2006 [http://www.espacestemps.net/articles/lrsquohypothese-de-lrsquohabiter-poly-topiquepratiquer-les-lieux-geographiques-dans-les-societes-a-individus-mobiles/]. 
5. Voir par exemple : Mathis зтоск (dir.), Habiter, Travaux de l'Institut géographique de Reims, vol. 29-30, n 115-118, 2003-2004 ; Olivier LAZZAROTTI, Béatrice COLLIGNON et Philippe PELLETIER (dir.), Habiter : mots et regards croisés, Annales de géographie, $\mathrm{n}^{\circ}$ 704, 2015.

6. Voir par exemple : Olivier LAZZAROTTI et Régis GUYON (dir.), Habiter l'école, lieu ouvert, lieu fermé ?, Diversité, n 179, 2015; Olivier LAzZAROTTI, Habiter le Monde, Documentation photographique, $\mathrm{n}^{\circ} 8100,2014$.

7. Voir par exemple: Jacques LÉVY, Le tournant géographique. Penser l'espace pour lire le monde, "Mappemonde », Paris : Belin, 1999 ; Michel Lussault, L'avènement du Monde. Essai sur l'habitation humaine de la Terre, coll. « La couleur des idées », Seuil, 2013.

\section{RÉSUMÉS}

En s'appuyant sur le renouvellement des études géographiques sur le tourisme, s'est imposée, dans le courant des années 1990, la nécessité d'inventer un nouveau concept capable de prendre acte et de rendre compte des singularités des « sociétés à habitants mobiles » dans un Monde en bouleversement. Nommant la dimension géographique de l'humanité, le concept d'« habiter » est aujourd'hui retourné vers son champ de départ, pour dévoiler l'invisible du tourisme : celui qui fait de chaque touriste un(e) habitant(e) et de tous des cohabitants. Cela conduit alors à faire l'hypothèse que le "régime d'habiter" touristique est à l'un des fondements du Monde contemporain.

On the basis of the renewal of geographical studies on tourism, it became necessary in the course of the 1990s to invent a new concept capable of recognizing and reporting on the singularities of "societies with mobile inhabitants". Naming the geographical dimension of humanity, the concept of "dwelling" is now returned to its starting field, to unveil the invisible of the tourism: the one that makes of each tourist an inhabitant and of all of them cohabitants. This leads, then, to the hypothesis that the tourist "regime of dwelling" is at one of the foundations of the contemporary world.

\section{INDEX}

Mots-clés : tourisme, monde, habiter, habitant, cohabitant

Keywords : tourism, world, dwelling, inhabitants, cohabitants

\section{AUTEUR}

\section{OLIVIER LAZZAROTTI}

Professeur de géographie, Université de Picardie-Jules-Verne 